\title{
Arzt-Patienten-Kommunikation
}

\section{Verhindern Sie „Aufklärungsschäden“!}

\author{
Zeitdruck und die Angst, verklagt zu werden, führen häufig dazu, dass Ärzte ihre Aufklä- \\ rungsgespräche eher an die juristischen Anforderungen anpassen als an die Bedürfnisse \\ der Patienten. Dies kann aber verheerende Auswirkungen haben.
}

\begin{abstract}
_ Als die 75-Jährige mit Abwehrspannung in die Notaufnahme kam und freie abdominale Luft nachgewiesen wurde, war der Fall klar: Sie musste notfallmäBig operiert werden. Doch nachdem sie vom Anästhesisten über Art und Durchführung, Folgen, Risiken, Dringlichkeit, Eignung, Erfolgsaussichten, Notwendigkeit und Alternativen der Narkose aufgeklärt wurde, entschied sie sich gegen die überlebenswichtige Operation.

„Die Patientin war nach der Aufklärung völlig verängstigt“", berichtete Prof. Martin Kreis, Direktor der Chirurgischen Klinik I der Berliner Charité, auf dem Chirurgenkongress in München.

Vor diesem Problem stehen Ärzte immer häufiger: Einerseits werden sie durch Zeitmangel oft dazu verleitet, $\mathrm{Pa}$ tienten in einem sehr kurzen Zeitraum
\end{abstract}

Den Patienten beim Aufklärungsgespräch dort abholen, wo er steht.

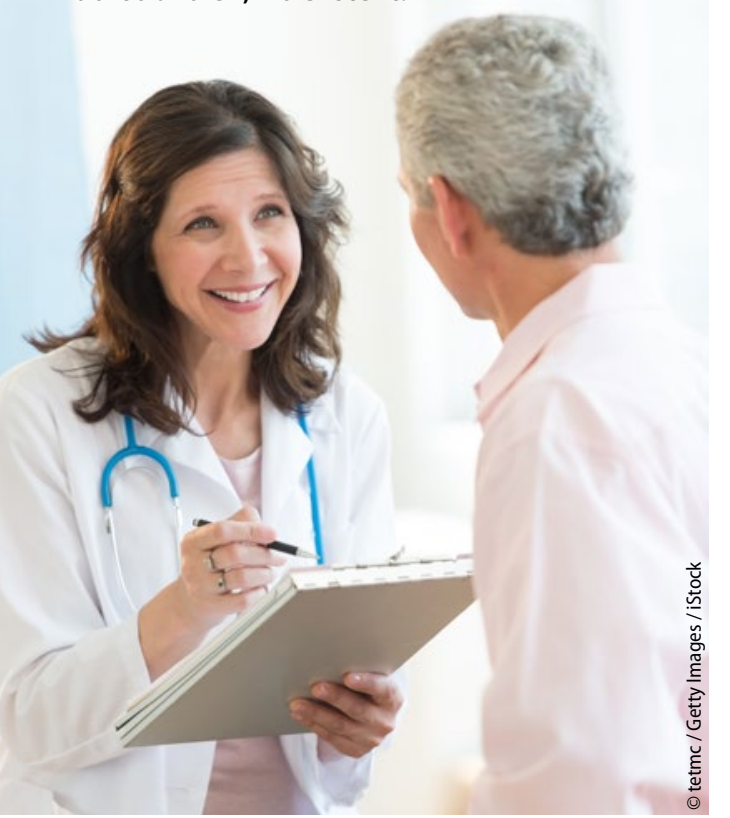

eine Fülle an komplexen medizinischen Sachverhalten „herunterzubeten“ und dies ausführlich zu dokumentieren, um sich rechtlich abzusichern, andererseits sollten sie aber auch sicherstellen, dass die Patienten durch das Gespräch die Bedeutung eines Eingriffs verstehen und entsprechend fähig sind, sich dafür oder dagegen zu entscheiden.

„Klärt man erbarmungslos auf und nimmt mehr Rücksicht auf die eigenen rechtlichen Risiken als auf die gesundheitlichen Risiken des Patienten, ist man aus forensischer Sicht auf der sicheren Seite“, erklärte der Fachanwalt für Medizinrecht und Facharzt für Chirurgie Dr. Helge Hölzer. „Doch niemand macht sich darüber Gedanken, ob der Patient wirklich von dieser Form der Aufklärung profitiert." Dadurch entsteht das Risiko, dass Patienten eine wichtige Behandlung aus Angst vor den Risiken und Nebenwirkungen ablehnen.

\section{Erektionsstörung durch Aufklärung} Interessanterweise beeinflussen die Wortwahl und die Art, wie aufgeklärt wird, nicht nur die Entscheidung des Patienten, sondern auch potenzielle $\mathrm{Ne}$ benwirkungen. So konnte z. B. in einer italienischen Studie gezeigt werden, dass die Art und das Ausmaß der Aufklärung Einfluss auf die Nebenwirkungsrate von Betablockern hat [1].

Wurden die Probanden nicht darüber informiert, welches Medikament sie einnahmen, traten Erektionsstörungen mit einer Häufigkeit von 3,1\% auf. Klärten die Behandler die Probanden darüber auf, dass sie Atenolol bekamen, ohne den Patienten weitere Informationen dazu zu geben, stieg der Anteil auf 15,6\%. Sagten die Ärzte hingegen: „Es können
Erektionsstörungen auftreten, aber das ist selten", ohne auf das individuelle Risiko des Probanden einzugehen, lag die Häufigkeit bei 31,2\% [1]. Begünstigende Faktoren für solche Noceboeffekte entstehen laut Kreis u. a.,

- wenn man dem Patienten die Aufklärung aufdrängt,

- wenn er damit überrascht wird,

- bei Zeitmangel,

- bei mangelnder Empathie,

- bei einer drastischen Wortwahl,

- bei mangelhafter therapeutischer Beziehung,

- infolge von Angst und

- wenn Fachjargon und unverständliche Worte verwendet werden.

\section{Tipps für das Patientengespräch}

„Man kann durch ein gutes Aufklärungsgespräch eine sehr gute Beziehung zwischen Patient und Therapeut herstellen", betonte Kreis. Dafür sei es wichtig, dass der Arzt folgende Punkte berücksichtigt:

- Er sollte seine Aufklärung an den Wissensstand des Patienten anpassen, - den Inhalt der Aufklärung in den Gesamtzusammenhang stellen,

- individuell darauf eingehen, wie das persönliche Risiko des einzelnen Patienten ist und

- das Aufklärungsgespräch so früh wie möglich führen.

Auch bei der 75-Jährigen mit Abwehrspannung hatte Kreis auf diese Weise Erfolg: Nach einem zusätzlichen Gespräch beruhigte sich die Patientin. Sie verstand schließlich, weshalb dieser Eingriff akut notwendig war und willigte ein.

Dr. med. Constance Jakob

\footnotetext{
- 134. Kongress der Deutschen Gesellschaft für Chirurgie,

24. März 2017, München
}

1. Silvestri A et al. Eur Heart J 2003;24:1928-32 